\title{
A TEST OF VE-THEORIES OF RISK AND \\ THE EFFECT OF THE CENTRAL LIMIT THEOREM
}

CLYDE H. COOMBS ${ }^{1}$ and JAMES N. BOWEN ${ }^{2}$

The University of Michigan, Department of Psychology, Ann Arbor, Mich. 48104, U.S.A.

\begin{abstract}
There are several theories of risk which indicate that risk could be a function only of variance and expectation. A transformation on odds or skewness was constructed which left the variance and expectation of a gamble unchanged. Perceived risk was clearly a function of this transformation as well as variance and expectation, even under multiple play in which the effect of the central limit theorem modifies the effect of skewness but it remains a relevant variable.
\end{abstract}

CoOmbs and Huang (1970) investigated the structure of perceived risk by proposing that certain transformations defined on gambles induce corresponding subjective transformations on the perceived risk of those gambles. They further proposed that the conjoint effect of those subjective transformations would have a particular polynomial form. This theory was tested by conjoint measurement methods (KRANTZ and TVERSKY, 1970), using two-outcome gambles of the form $g=(y, p, z)$, in which the outcome $y$ occurs with probability $p$ otherwise $z, y, \geqslant z$. The transformations on gambles which were used in the study were

$$
\begin{aligned}
& a(g)=(y+a, 1 / 2, z-a), \\
& b(g)=(y+b, 1 / 2, z+b), \\
& c(g)=(y, 1 / 2, z)^{(c)},
\end{aligned}
$$

where $a$ and $b$ are amounts of money and $c$ is a non-negative integer indicating the gamble is played $c$ times independently.

The transformation $a(g) \in A$ preserves expectation but directly in-

1 This research was supported by PHS grant HD-17844-02 and NSF grant GB15653. We wish to express our appreciation for helpful comments from David Krantz and J. E. Keith Smith.

2 Now at the University of Texas at Arlington. 
creases variance, expected loss, expected regret, the maximum loss, with any of which perceived risk might increase monotonically.

The transformation $b(g) \in B$ increases expectation and such changes as it makes in other variables would also tend to decrease perceived risk.

The transformation $c(g) \in C$ tends to multiply the joint effect of $a(g)$ and $b(g)$.

The theory proposed was that corresponding to the transformations $a(g), b(g)$, and $c(g)$ on a gamble there existed corresponding subjective transformations $\alpha, \beta$, and $\gamma$ on the perceived risk of the gamble, and that their joint effect was given by the so-called distributive model:

$$
R(g)=[\alpha(g)+\beta(g)] \gamma(g),
$$

where $R(g)$ is the riskiness of the gamble. The experimental results supported this polynomial over certain other alternative polynomials.

POLLATSEK and TVERSKY (1970) then provided an axiomatic theory of risk and showed that the above polynomial is compatible with it. One of the results of their axioms is that risk is a linear function of variance and expectation. The proof of this rests heavily on the central limit theorem which says that the probability that the outcome of any gamble will be in any given interval approaches that of a normal distribution with the same mean and variance, as $n$, the number of times the gamble is convoluted with itself (e.g., played $n$ times independently), approaches infinity and the outcomes are rescaled by multiplying by $1 / 1 / n$. Given this result and the fact that a normal distribution is completely characterized by its mean and variance those two parameters must be sufficient to measure the riskiness of a gamble that is not a normal distribution provided continuity exists in the risk function, which Pollatsek and Tversky assume.

This result implies that transformations on gambles which do not change a gamble's variance and expectation will leave their risk order invariant. An obvious possibility to be investigated is to change the probability and at the same time make corrective changes in the variance and expectation, so that only the odds are changed. For gambles of the form $g=(a, 1 / 2,-a)$, which have expectation zero and variance $a^{2}$, the transformation:

$$
p(g)=(a / q / p, p,-a \mid p / q)
$$

where $p(g) \in P$ and $0<p<1$ will change the odds or skewness and will leave the expectation and variance unchanged. The further transforma- 
tions $b(g)$ and $c(g)$ may then be performed. If this transformation $p(g)$, does not leave the risk order invariant, then the odds make a contribution to perceived risk with variance and expectation held constant.

If this transformation is found to be relevant to perceived risk then it is of interest whether or not the effect can be captured by a simple change in the polynomial of eq. (4). Also if this $p(g)$ transformation is a relevant variable, then one can independently test the effect of the central limit theorem by comparing the effect of skewness on perceived risk at two levels of $C$.

\section{METHOD}

\subsection{Subjects}

The $S$ s were paid student volunteers at the University of Michigan, 18 men and 9 women. Each $S$ was run individually.

\subsection{Design}

One set of games was generated in a $3 \times 3 \times 3$ matrix by independently varying expected value, variance, and probability of winning. The three levels of probability used were $1 / 4,1 / 2,3 / 4$. The games at $p=1 / 2$ under single play $(c=1)$ are presented in table 1 in dollars as units.

TABLE 1

Games for $p=1 / 2, c=1$.

\begin{tabular}{|c|c|c|c|}
\hline$a^{\prime \prime}=4.00$ & $(4.00,1 / 2,-4.00)$ & $(4.40,1 / 2,-3.60)$ & $(4.80,1 / 2,-3.20)$ \\
\hline$A a^{\prime}=3.00$ & $(3.00,1 / 2,-3.00)$ & $(3.40,1 / 2,-2.60)$ & $(3.80,1 / 2,-2.20)$ \\
\hline$a=2.00$ & $(2.00,1 / 2,-2.00)$ & $(2.40,1 / 2,-1.60)$ & $(2.80,1 / 2,-1.20)$ \\
\hline
\end{tabular}

To generate the games for the other levels of $P$, the transformation $p(g)$, (eq. (5)), was used on the games in the first column in table 1 and then the $b(g)$ transformation was applied to obtain the other two levels of expected risk.

The games at $p=1 / 4$ and at $p=3 / 4$ are presented in tables 2 and 3 respectively. 
TABle 2

Games for $p=1 / 4, c=1$.

\begin{tabular}{r|c|c|c|}
$a^{\prime \prime}=4.00$ & $(6.90,1 / 4,-2.30)$ & $(7.30,1 / 4,-1.90)$ & $(7.70,1 / 4,-1.50)$ \\
$A a^{\prime}=3.00$ & $(5.19,1 / 4,-1.73)$ & $(5.59,1 / 4,-1.33)$ & $(5.99,1 / 4,-.93)$ \\
\cline { 2 - 5 }$a=2.00$ & $(3.45,1 / 4,-1.15)$ & $(3.85,1 / 4,-.75)$ & $(4.25,1 / 4,-.35)$ \\
\hline \multicolumn{3}{c}{$b=0$} & $b^{\prime}=40$ \\
$B$
\end{tabular}

TABLE 3

Games for $p=3 / 4, c=1$.

\begin{tabular}{rl|c|c|c|}
$a^{\prime \prime}=4.00$ & $(2.30,3 / 4,-6.90)$ & $(2.70,3 / 4,-6.50)$ & $(3.10,3 / 4,-6.10)$ \\
\hline$a^{\prime}=3.00$ & $(1.73,3 / 4,-5.19)$ & $(2.13,3 / 4,-4.79)$ & $(2.53,3 / 4,-4.39)$ \\
\hline & $(1.15,3 / 4,-3.45)$ & $(1.55,3 / 4,3.05)$ & $(1.95,3 / 4,-2.75)$ \\
\hline & $b=0$ & $b^{\prime}=40$ & $b^{\prime \prime}=80$ \\
& $B$ &
\end{tabular}

This set of $3 \times 3 \times 3$ games is called the single play set. A second set of games was generated from the first set by a multiple play transformation $(y, p, z)^{(c)}$, with $C=24$.

\subsection{Stimuli}

The games were printed on cards and embedded in laminated plastic. The manner in which single play games were displayed is illustrated in fig. 1.
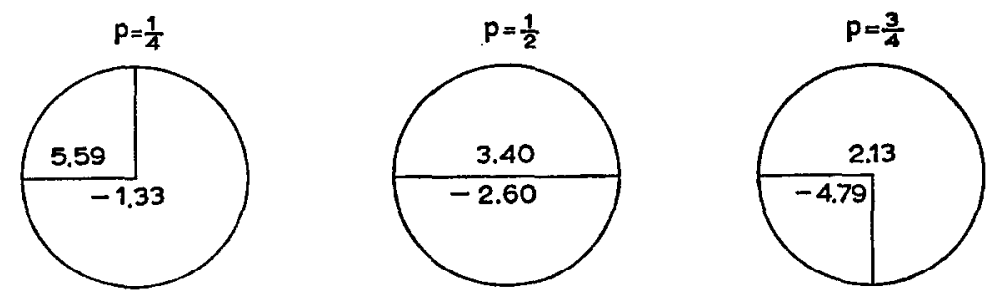

Fig. 1. Types of display for single play games.

The multiple play games were presented as 25 -outcome games, each to be played once, and displayed in two ways to the $S$ s. On one side of each stimulus card was a graph of the appropriate discrete probability distribution over money; on the other side was a list of the 25 possible out- 


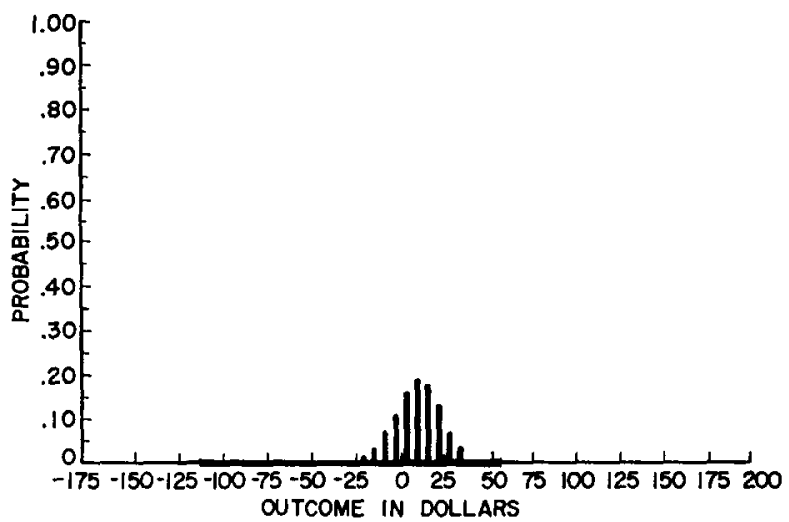

Fig. 2. One side of a stimulus card.

TABLE 4

The other side of a stimulus card.

\begin{tabular}{|c|c|}
\hline Outcome & Probability \\
\hline$\$ 50.60$ & 0.001 \\
\hline 43.70 & 0.007 \\
\hline 36.80 & 0.031 \\
\hline 29.90 & 0.075 \\
\hline 23.00 & 0.132 \\
\hline 16.10 & 0.175 \\
\hline 9.20 & 0.185 \\
\hline 2.30 & 0.160 \\
\hline$-\quad 4.60$ & 0.113 \\
\hline-11.50 & 0.067 \\
\hline-18.40 & 0.033 \\
\hline-25.30 & 0.014 \\
\hline-32.30 & 0.004 \\
\hline-39.10 & 0.0013 \\
\hline-46.00 & 0.00035 \\
\hline-52.90 & 0.000077 \\
\hline-59.80 & 0.000015 \\
\hline-66.70 & 0.0000023 \\
\hline-73.60 & 0.0000003 \\
\hline-80.50 & 0.00000003 \\
\hline-87.40 & 0.0000000026 \\
\hline-94.30 & 0.00000000017 \\
\hline-101.20 & 0.0000000000075 \\
\hline-108.10 & 0.00000000000023 \\
\hline-115.00 & 0.000000000000003 \\
\hline
\end{tabular}


comes with their respective probabilities. Examples of the two sides of a stimulus card are given in fig. 2 and table 4 in which the single play game from which this multiple play game was generated was $(2.13,3 / 4,-4.79)$. Outcomes with very low probability were suggested by the broad base in the figure. On the actual stimulus card the distribution was drawn in red. The $S$, of course, was informed that the actual outcomes and probabilities were displayed on the reverse of the card.

\subsection{Procedure}

Each $S$ came for three sessions. During the first session, the $S$ rank ordered each set of games in terms of their perceived riskiness. A coin toss determined which set was ranked first. The second and third sessions were a replication of the first. The following version of a method of accumulation was used to obtain the rank orders: The $S$ was presented with three games, selected for easy discrimination, and told to order them according to how risky they appeared. A fourth gamble, randomly selected, was then presented and $S$ was told to place it relative to the first three. A fifth game was then placed relative to the first four, etc., until all 27 games were ranked. The $S$ was then asked to check the final ordering and to correct any errors of judgment he might have made.

In judging the multiple play set of games, $S$ was free to use either or both sides of the stimulus card.

\section{RESULTS}

\subsection{Consistency}

The $S$ s were rank ordered in consistency based on their average $\tau$ between the three replications on each set of stimuli. Hence, the $S$ s were ranked in consistency on the set of single play games and independently ranked in consistency on the set of multiple play games. The $\tau$ coefficient between these two rankings in consistency was itself 0.339 . This low correlation led us to keep the consistency orderings distinct for the two sets of games. In certain further analyses below, then, $S$ s are divided into three subgroups of nine $S \mathrm{~s}$ in each, $\mathrm{H} \equiv$ highest consistency, $\mathrm{M} \equiv$ medium consistency, and $\mathrm{L} \equiv$ lowest consistency, based on their consistency ranking on the set of single play games and again based on their consistency ranking on the set of multiple play games.

The average $\tau$ for each subgroup for each of the sets of games is given in table 5 . 
TABLE 5

Consistency level $(\overline{\boldsymbol{\tau}})$ of each subgroup for each set of stimuli.

\begin{tabular}{|c|c|c|c|c|}
\hline \multicolumn{4}{|c|}{ Subgroup } & \multirow[b]{2}{*}{ Total group } \\
\hline & $\mathbf{H}$ & $\mathbf{M}$ & L & \\
\hline$c=1$ & 0.922 & 0.763 & 0.340 & 0.678 \\
\hline$c=24$ & 0.866 & 0.719 & 0.478 & 0.688 \\
\hline
\end{tabular}

\subsection{Effects of odds}

If the effect of a transformation from the set $P$ is irrelevant to perceived risk or relevant but independent of the effect of variance and expectation, then the ordering induced on $A \times B$ with the levels of $P$ and $C$ fixed should be independent of the level of $P$ (cf. KRANT $\angle$ and TVERSKY, 1970; COOMBS and Huang, 1970). Furthermore, the ordering induced on $P$ with the levels of $A \times B$ and of $C$ fixed should be degenerate if $P$ is an irrelevant variable or should be invariant over the levels of $A \times B$ if the contribution of $P$ is independent of the joint effect of $A \times B$.

These scale-free tests permit us to diagnose whether the effect of the change in probability has been emasculated by the corrective changes in variance and expectation or whether, if an effect persists, it is independent of the effect of variance and expectation.

Following the notation introduced in Coombs and Huang (1970) $A \times B ; P: c=1$ signifies a test of whether the ordering induced on $A \times B$ with $P$ and $C$ fixed ( $C$ fixed at $c=1$ ) is independent of the level at which $P$ is fixed.

To make this test requires comparing the orderings of $S$ of the games in each of the three tables (tables 1, 2 and 3), i.e., three orderings of nine elements. We used as the criterion level for judging whether a test was satisfied the average $\tau$ that would be obtained if there were exactly one pairwise reversal in one of the orderings of nine elements, here a $\bar{\tau}$ of 0.852 .

The number of $S$ s who satisfy each of the tests at the indicated criterion level are given in table 6.

Lowering the criterion level for the tests of the independence of $A \times B$ with respect to $P$ from exactly one pairwise reversal in one of the orderings to exactly three independent pairwise reversals in one of the orderings lowers $\bar{\tau}$ to 0.889 but makes no essential change in the results: the 5, 2,0 in the first column become 6,3,0 respectively, and the $0,0,0$ of the second column become 1, 0, 0 for the $H, M$, and $L$ subgroups. 
TABLE 6

Tests of the independence of $A \times B$ and $P$.

\begin{tabular}{ccccc}
\hline & $\begin{array}{c}A \times B ; P: c=1 \\
\overline{\boldsymbol{\tau}} \geqslant 0.963\end{array}$ & $\begin{array}{c}A \times B ; P: c=24 \\
\overline{\boldsymbol{\tau}} \geqslant 0.963\end{array}$ & $\begin{array}{c}P ; A \times B: c=1 \\
\bar{\tau} \geqslant 0.850\end{array}$ & $\begin{array}{c}P ; A \times B: c=24 \\
\overline{\boldsymbol{\tau}} \geqslant 0.850\end{array}$ \\
& & Test $^{*}$ & \\
\hline H & 5 & 0 & 9 & 9 \\
M & 2 & 0 & 7 & 9 \\
L & 0 & 0 & 6 & 7 \\
Total & 7 & 0 & 22 & 25 \\
\hline
\end{tabular}

* Cell entries are the number of $S$ s out of nine who satisfy the test.

The first two tests in table 6 indicate that $P$ is still a relevant variable in spite of having no effect on variance and expectation, especially under multiple play. This is confirmed by the last two tests in table 6 which indicate that the contribution of the $P$ transformation to pereived risk is independent of the contribution from the joint effect of $A \times B$.

\subsection{Test of the effect of the central limit theorem}

Table 7 contains the mean rank of the nine games at each level of $P$ and $C$ for each $S$. The mean rank of the 27 games at each level of $C$ is, of course 14 , so the sum of each row of the table is a constant 42 . At each level of $C$ the mean absolute deviation about 14 was calculated and these two quantities were compared for each $S$. The larger this deviation the greater the effect of the $P$ transformation on risk.

The mean absolute deviation was greater for $c=1$ than for $c=24$ for 23 of the $27 \mathrm{Ss}$. A sign test for matched pairs yields a $z=3.46$ which is significant $(p<0.001)$. This result is consistent with the notion that multiple play assuages the influence of probability on risk perception.

In addition to the general effect of multiple play on the influence of skewness on risk there is within the subject an effect that is independent and which is of interest for descriptive purposes primarily. At $c=1$ odds for or against may differentially effect risk and then multiple play may have a further differential effect. We shall speak of a game with $p=3 / 4$ played once or the same game under multiple play as a negatively skewed game (see fig. 2 for an example). Similarly a game with $p=1 / 4$ or a game generated from such a game will be spoken of as a positively skewed bet.

The basic data are presented in table 8 . As may be seen, at single play about two-thirds of the $S$ s regard unfavorable odds as riskier (with ex- 
TABLE 7

Mean rank * of the nine games at each level of $P$ and $C$ for each $S$.

\begin{tabular}{|c|c|c|c|c|c|c|c|c|c|}
\hline \multirow[t]{2}{*}{$S \#$} & \multirow[t]{2}{*}{$C$} & \multicolumn{3}{|c|}{$P$} & \multirow[t]{2}{*}{$S \#$} & \multirow[t]{2}{*}{$C$} & \multicolumn{3}{|c|}{$P$} \\
\hline & & $3 / 4$ & $1 / 2$ & $1 / 4$ & & & $3 / 4$ & $1 / 2$ & $1 / 4$ \\
\hline \multirow[t]{2}{*}{1} & 1 & 10.78 & 16.67 & 14.55 & 15 & 1 & 13.00 & 14.00 & 15.00 \\
\hline & 24 & 16.67 & 14.33 & 11.00 & & 24 & 20.67 & 13.33 & 8.00 \\
\hline \multirow[t]{2}{*}{2} & 1 & 12.44 & 18.56 & 11.00 & 16 & 1 & 21.56 & 14.56 & 5.88 \\
\hline & 24 & 14.11 & 12.11 & 15.78 & & 24 & 16.22 & 15.11 & 10.67 \\
\hline \multirow[t]{2}{*}{3} & 1 & 14.77 & 16.88 & 10.35 & 17 & 1 & 5.00 & 16.33 & 20.67 \\
\hline & 24 & 17.00 & 14.00 & 11.00 & & 24 & 14.44 & 15.56 & 13.00 \\
\hline \multirow[t]{2}{*}{4} & 1 & 6.00 & 15.00 & 21.00 & 18 & 1 & 5.00 & 14.00 & 23.00 \\
\hline & 24 & 13.44 & 14.00 & 14.56 & & 24 & 15.44 & 14.56 & 12.00 \\
\hline \multirow[t]{2}{*}{5} & 1 & 5.00 & 14.00 & 23.00 & 19 & 1 & 5.00 & 14.00 & 23.00 \\
\hline & 24 & 19.90 & 13.44 & 8.66 & & 24 & 23.00 & 14.00 & 5.00 \\
\hline \multirow[t]{2}{*}{6} & 1 & 23.00 & 14.00 & 5.00 & 20 & 1 & 5.00 & 14.00 & 23.00 \\
\hline & 24 & 11.00 & 8.33 & 22.67 & & 24 & 15.67 & 14.78 & 11.55 \\
\hline \multirow[t]{2}{*}{7} & 1 & 23.00 & 14.00 & 5.00 & 21 & 1 & 17.78 & 15.33 & 8.89 \\
\hline & 24 & 15.00 & 14.44 & 12.56 & & 24 & 18.22 & 14.22 & 9.56 \\
\hline \multirow[t]{2}{*}{8} & 1 & 5.00 & 14.00 & 23.00 & 22 & 1 & 5.00 & 16.00 & 21.00 \\
\hline & 24 & 13.11 & 15.55 & 13.33 & & 24 & 15.33 & 14.89 & 11.78 \\
\hline \multirow[t]{2}{*}{9} & 1 & 10.33 & 17.00 & 14.67 & 23 & 1 & 5.00 & 14.00 & 23.00 \\
\hline & 24 & 17.33 & 14.55 & 10.12 & & 24 & 22.44 & 14.33 & 5.23 \\
\hline \multirow[t]{2}{*}{10} & 1 & 20.89 & 12.33 & 8.78 & 24 & 1 & 5.00 & 14.00 & 23.00 \\
\hline & 24 & 14.67 & 14.55 & 12.78 & & 24 & 14.67 & 16.56 & 10.77 \\
\hline \multirow[t]{2}{*}{11} & 1 & 19.44 & 14.33 & 8.23 & 25 & 1 & 5.00 & 14.00 & 23.00 \\
\hline & 24 & 14.55 & 13.89 & 11.56 & & 24 & 12.77 & 13.44 & 15.79 \\
\hline \multirow[t]{2}{*}{12} & 1 & 5.00 & 16.56 & 20.44 & 26 & 1 & 11.33 & 15.00 & 15.67 \\
\hline & 24 & 14.22 & 12.56 & 15.22 & & 24 & 16.22 & 14.78 & 11.00 \\
\hline \multirow[t]{2}{*}{13} & 1 & 5.00 & 14.00 & 23.00 & 27 & 1 & 14.44 & 16.88 & 10.68 \\
\hline & 24 & 17.78 & 16.11 & 8.11 & & 24 & 16.44 & 14.33 & 11.23 \\
\hline \multirow[t]{2}{*}{14} & 1 & 16.88 & 14.55 & 10.57 & & & & & \\
\hline & 24 & 13.00 & 13.67 & 15.33 & & & & & \\
\hline
\end{tabular}

* The higher the rank the riskier the game.

pectation and variance controlled) but under multiple play this relation appears not to hold and may even be reversed. There are only $7 \mathrm{Ss}$ (the main diagonal) for whom the relation of risk to skewness is the same under single and multiple play. For 20 of the $S$ s this relation was reversed.

A count made from table 7 indicates that there were $21 \mathrm{Ss}$ for whom negative skewness got relatively riskier under multiple play as against only 10 for whom positive skewness got relatively riskier. There were 
TABLE 8

Effect of multiple play on the relation of skewness to perceived risk.

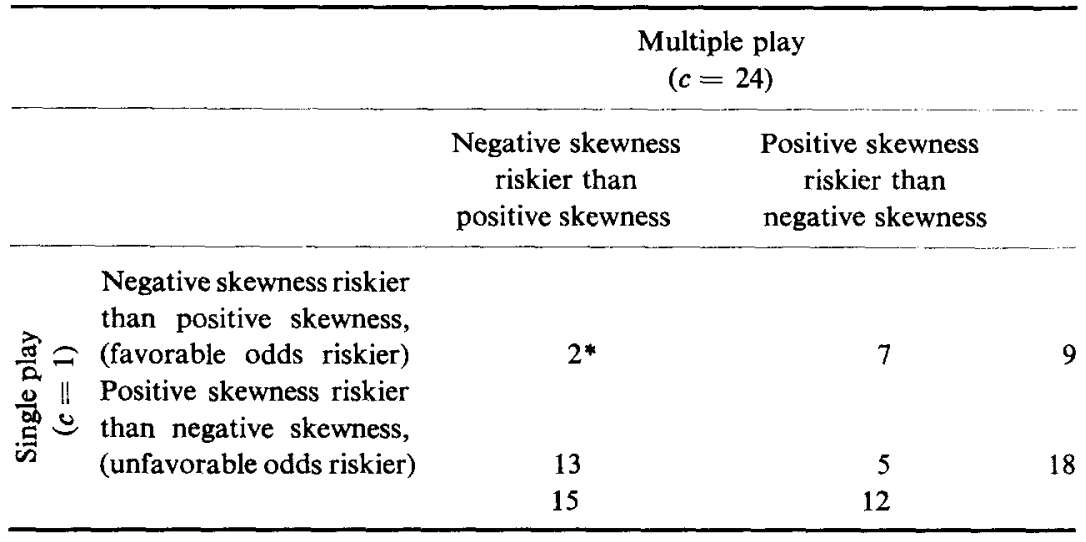

* Number of $S \mathrm{~s}$.

also four $S$ s, for whom the effect of multiple play was to make any skewed games riskier relative to the effect on symmetric games.

These relations could reflect inherent interactions which the methods of conjoint measurement are well suited to test. This analysis is discussed next.

\subsection{Diagnostic properties for poly nomials}

It might be conjectured that the four variables with which this study is concerned, $A, B, P$ and $C$ induce four corresponding transformations on perceived risk whose joint effect can be represented by a simple polynomial, a simple polynomial being one obtained by either the successive addition or multiplication of the four variables in some sequence.

There are, altogether, 48 possible polynomials of that kind and this study is too limited for complete diagnosis by conjoint measurement methods, particularly in view of the possibility that one of the variables $(p)$ may generate sign-dependencies. It is possible, however, to draw some inferences so the results of the tests that could be made are presented here in tables 9 and 10 .

The tables report the number of $S \mathrm{~s}$ out of a possible nine in each cell who satisfy the test in question at the level indicated by the $\bar{\tau}$ at the top of each column. The higher $\bar{\tau}$ reflects exactly one pairwise reversal in one ordering of the three orderings of $\bar{\tau}$ elements. The lower $\bar{\tau}$ reflects exactly 
TABLE 9

Tests* of diagnostic properties for polynomials under single play, $c=1$.

\begin{tabular}{lccccccccc}
\hline \multicolumn{1}{c}{ Interplane tests } \\
& \multicolumn{1}{c}{$\boldsymbol{A} \times B ; P$} & $P ; A \times B$ & $A \times P ; B$ & $B ; A \times P$ & $B \times P ; A$ & $A ; B \times P$ \\
\hline & $\bar{\tau} \geqslant$ & $\bar{\tau} \geqslant$ & $\bar{\tau} \geqslant$ & $\bar{\tau} \geqslant$ & $\bar{\tau} \geqslant$ & $\bar{\tau} \geqslant$ & $\bar{\tau} \geqslant$ & $\bar{\tau} \geqslant$ & $\bar{\tau} \geqslant$ \\
& 0.963 & 0.889 & 0.852 & 0.963 & 0.889 & 0.852 & 0.963 & 0.889 & 0.852 \\
\hline H & 5 & 6 & 9 & 7 & 8 & 6 & 1 & 6 & 7 \\
M & 2 & 3 & 7 & 1 & 5 & 6 & 0 & 2 & 6 \\
L & 0 & 0 & 6 & 0 & 0 & 1 & 0 & 0 & 1 \\
\hline Total & 7 & 9 & 22 & 8 & 13 & 13 & 1 & 8 & 14 \\
\hline
\end{tabular}

Intraplane tests

\begin{tabular}{lcccccc} 
& $A ; P: B$ & $P ; A: B$ & $A ; B: P$ & $B ; A: P$ & $B ; P: A$ & $P ; B: A$ \\
\hline i $\geqslant 0.852$ & $\bar{\tau} \geqslant 0.852$ & $\bar{\tau} \geqslant 0.852$ & $\bar{\tau} \geqslant 0.852$ & $\bar{\tau} \geqslant 0.852$ & $\overline{\boldsymbol{\tau}} \geqslant 0.852$ \\
\hline M & 7 & 9 & 9 & 6 & 7 & 9 \\
L & 6 & 7 & 7 & 6 & 6 & 7 \\
\hline Total & 1 & 6 & 1 & 1 & 1 & 6 \\
\hline
\end{tabular}

* Cells report the number of $S$ s out of nine who satisfy the test in question at the level indicated.

three independent pairwise reversals in one of the three orderings on nine elements.

Because our fourth variable, $C$, is represented at only two levels and there are no data on orderings induced on $C$ we will only examine the simple polynomials involving the functions corresponding to the transformations $A, B$, and $P$. These models in three variables are as follows:

$$
\begin{array}{ll}
r_{1}+r_{2}+r_{3} & \text { additive model, } \\
\left(r_{1}+r_{2}\right) r_{3} & \text { distributive model, } \\
r_{1} r_{2}+r_{3} & \text { dual distributive model, } \\
r_{1} r_{2} r_{3} & \text { multiplicative model. }
\end{array}
$$


TABLE 10

Tests* of diagnostic properties for polynomials under multiple play, $c=24$.

\begin{tabular}{cccccccccc}
\hline \multicolumn{10}{c}{ Interplane tests } \\
& $A \times B ; P$ & $P ; A \times B$ & $A \times P ; B$ & $B ; A \times P$ & $B \times P ; A$ & $A ; B \times P$ \\
\hline & $\bar{\tau} \geqslant$ & $\vec{\tau} \geqslant$ & $\bar{\tau} \geqslant$ & $\bar{\tau} \geqslant$ & $\bar{\tau} \geqslant$ & $\bar{\tau} \geqslant$ & $\bar{\tau} \geqslant$ & $\bar{\tau} \geqslant$ & $\bar{\tau} \geqslant$ \\
& 0.963 & 0.889 & 0.852 & 0.963 & 0.889 & 0.852 & 0.963 & 0.889 & 0.852 \\
\hline H & 0 & 1 & 9 & 1 & 6 & 3 & 1 & 4 & 2 \\
M & 0 & 0 & 9 & 0 & 2 & 3 & 1 & 3 & 1 \\
L & 0 & 0 & 7 & 0 & 2 & 5 & 0 & 2 & 2 \\
\hline Total & 0 & 1 & 25 & 1 & 10 & 11 & 2 & 9 & 5 \\
\hline
\end{tabular}

Intraplane tests

\begin{tabular}{lcccccc} 
& $A ; P: B$ & $P ; A: B$ & $A ; B: P$ & $B ; A: P$ & $B ; P: A$ & $P ; B: A$ \\
\cline { 3 - 4 } & $\bar{\tau} \geqslant 0.852$ & $\bar{\tau} \geqslant 0.852$ & $\bar{\tau} \geqslant 0.852$ & $\bar{\tau} \geqslant 0.852$ & $\bar{\tau} \geqslant 0.852$ & $\bar{\tau} \geqslant 0.852$ \\
\hline H & 3 & 9 & 2 & 2 & 2 & 9 \\
M & 1 & 9 & 1 & 3 & 3 & 9 \\
L & 4 & 7 & 2 & 5 & 5 & 7 \\
\hline Total & 8 & 25 & 5 & 11 & 11 & 25 \\
\hline
\end{tabular}

* Cells report the number of $S$ s out of nine who satisfy the test in question at the level indicated.

For simplicity of exposition we shall use the letters $A, B, C$, and $P$ in particular polynomials to represent the subjective functions on perceived risk induced by the corresponding mathematical transformations on games.

If a single variable is mutually independent with the joint effect of the remaining variables it is called semiadditive. We see from table 9 that $B \times P$ is not independent of $A$ at a stringent level (only $1 S$ out of 27) and even at a weaker level only about $30 \%$ of the $S$ s (8 out of 27) satisfy independence so we conclude that $B \times P$ and $A$ are not mutually independent and so $A$ is not semiadditive.

The additive model requires that each of the three variables be semiadditive so it may be rejected. There remain the possibilities th: there are two, one, or zero semiadditive factors.

If there are exactly two semiadditive $f_{c}$ ors then none of the four models is possible. 
If there is exactly one it must be $P$ or $B$. If $B$ is the semiadditive factor then $A P+B$ or $(A+P) B$ are possibilities. In either case $A$ and $P$ should be mutually interdependent and we see from the bottom half of table 10 that this appears to be the case.

If $P$ is the semiadditive factor then $A B+P$ or $(A+B) P$ are possibilities. In either case $A$ and $B$ should be mutually independent and we see from the bottom half of table 9 that they appear to be.

If there are no semiadditive factors then the distributive and the multiplicative model are possibilities. The multiplicative model may be rejected because every factor must be at least mutually sign-dependent and, as noted in rejecting the additive model, $A$ does not satisfy this condition. For the distributive model with no semiadditive factors there must be exactly one which is at least mutually sign-dependent and the remaining pair must be mutually independent. For these three variables, then, with $C$ fixed at $c=1$ the three polynomials that are not clearly eliminated are $A P+B,(A+B) P$, and $A B+P$.

If we turn to table 10 , with $C$ fixed at $c=24$ it is clear that there are no scmiadditive factors, so only $(A+B) P$ remains a possibility. But then $A$ and $B$ must be mutually independent and the bottom half of table 10 (columns 3 and 4) leaves that prospect very suspect.

\section{Discussion}

It is clear from this experiment that varying the odds without changing the variance or expectation still causes substantial changes in perceived risk. As a consequence any theory that requires risk to be a function solely of variance and expectation is violated. We note that the effect of odds is especially marked under multiple play.

It should be pointed out that the substantial effect observed here of the central limit theorem on perceived risk is obtained under display conditions in which the distribution obtained under multiple play is explicitly presented. Whether these same results would have been obtained if a 2-outcome game were presented with the information that it was to be played 24 times is in serious doubt. In the case of a few $S \mathrm{~s}$, after the experiment was concluded, some pairs of 2-outcome games were presented and $S$ was asked to judge which was riskier if the games were to be played 24 times. Almost invariably the $S$ s responded the same as they did to the games under single play. This is very clearly not what happened when they were presented with complete information about the effect of playing 24 times. 
Such a difference could reflect a failure in mental arithmetic, uninformed biases, or a real psychological difference in perceived risk in that if it can happen all at once it has a different riskiness than if it happens in successive stages like a random walk.

If all simple 4-variable polynomials are rejected there are at least two possibilities to consider. One possibility is that the effect of a given mathematical transformation on a game may be mediated by more than one psychological transformation on risk. For example, the effect of the transformation $P$ may be mediated by one function interacting with that for the $A$ transformation and another function interacting with that for the $B$ transformation, say $P^{\prime}$ and $P^{\prime \prime}$, in which case the polynomial could take the form $A P^{\prime}+B P^{\prime \prime}$, a form not tested in this experiment.

A second possibility is that the transformation $P$ utilized here is not the one best suited for combining with $A, B$, and $C$ in a simple polynomial. Alternative transformations which effect neither expectation nor variance or exactly one could be more suitable for the purpose.

(Accepted September 14, 1970.)

\section{REFERENCES}

Coomes, C. H. and L. C. Huang, 1970. Polynomial psychophysics of risk. Journal of Mathematical Psychology 7, 317-338.

Krantz, D. H. and A. Tversky, in press. Conjoint measurement analysis of composition rules in psychology. Michigan Mathematical Psychology Program Technical Report.

Pollatsek, A. and A. TVERSKy, in press. A theory of risk. Journal of Mathematical Psychology. 